\title{
Calling all users of Verbal Behavior
}

\author{
Kent R. Johnson \\ Morningside Learning Center
}

The following is a list of all college bookstores that have stocked Skinner's Verbal Behavior in the last four years. Presumably they stock the book because one or more professors use the book in one or more courses. If you are the professor who has ordered the book through one of the bookstores listed below, please let us know. We would like to get some information from all users about the courses that they teach. Perhaps more will be encouraged to adopt the book if some well tested ideas for use are made available. Surely we will all improve our courses with ideas from others.

Awards go to Iowa for stocking Verbal Behavior in three cities!

University of Florida, Gainsville, FL.

Libreria Internacional, Mexico

Western Campus Bookstore, Kalamazoo, MI. ASUN Bookstore, Reno, NV.

Georgia Tech Bookstore, Atlanta, GA.

Central College of Iowa, Pella, IW

The Bookstore, Las Vegas, NV

Teachers College Bookstore, New York, NY University Book Store, Salt Lake City, UT VPI Facilities, Inc. Blacksburg, VA.
International Book Distributers, Herts, HP2 4RG, England

Prentice-Hall SE Asia LD, Singapore, Malaya University of California, La Jolla, CA.

Georgetown University Bookstore, Washington, D.C.

Georgia Bookstore, Inc., Atlanta, GA.

University Bookstore, Des Moines, IW

Kansas Union Bookstore, Lawrence, KS.

Student Book Exchange, Columbus, $\mathrm{OH}$.

Associated Students Bookstore, Tucson, AZ.

Harvard Bookstore, Cambridge, MA.

Harvard University Bookstore, Cambridge, MA.

Newark Book Center, Newark, NJ.

University Book and Supply, Ceder Falls, IW

Nebraska Book Company, Lincoln, NB.

Vasser College Bookstore, Poughkeepsie, NY.

University of Pittsburgh, Pittsburgh, PA.

American University Campus Bookstore, Washington, DC.

Bill's Bookstore, Tallahassee, FL.

University of Texas at Arlington, Arlington, TX.

Utah State University, Logan UT.

University of Vermont, Burlington, VT.

Nueva Editorial, Mexico. 